\title{
CAUCHY'S ERROR REPRESENTATION OF LIDSTONE INTERPOLATING POLYNOMIAL AND RELATED RESULTS
}

\author{
G. Aras-Gazić, V. ČUlJak, J. PeČArić And A. Vukelić
}

\begin{abstract}
In this paper we consider the Cauchy's error representation of Lidstone interpolating polynomial and as a consequence the results concerning to the Hermite-Hadamard inequalities. Using these inequalities, we produce new exponentially convex functions. Also, we give several examples of the families of functions for which the obtained results can be applied.
\end{abstract}

Mathematics subject classification (2010): Primary 26D15; Secondary 26D07, 26 A51.

Keywords and phrases: Green function, $(2 n)$-convex function, Lidstone polynomial, Cauchy type mean value theorems, Hermite-Hadamard inequality, $n$-exponential convexity, exponential convexity, $\log$ convexity, means.

\section{REFERENCES}

[1] R. P. Agarwal, P. J. Y. Wong, Error Inequalities in Polynomial Interpolation and Their Applications, Kluwer Academic Publishers, Dordrecht/Boston/London, 1993.

[2] K. E. AtKinson, An Introduction to Numerical Analysis, 2nd ed., Wiley, New York, 1989.

[3] W. Ehm, M. G. Genton, T. Gneiting, Stationary covariance associated with exponentially convex functions, Bernoulli 9 (4) (2003), 607-615.

[4] J. JAKŠEtiĆ, J. PeČARIĆ, Exponential Convexity Method, J. Convex Anal. 20 (1) (2013), 181-197.

[5] S. KARLin, Total Positivity, Stanford Univ. Press, Stanford, 1968.

[6] S. KARLin, W. J. STUdDEN, Tchebycheff systems: with applications in analysis and statistics, Interscience, New York, 1966.

[7] J. PEČARIĆ, J. PERIĆ, Improvements of the Giaccardi and the Petrović inequality and related results, An. Univ. Craiova Ser. Mat. Inform., 39 (1) (2012), 65-75.

[8] J. E. PeČARIĆ, F. Proschan AND Y. L. TONG, Convex functions, partial orderings, and statistical applications, Mathematics in science and engineering 187, Academic Press, 1992.

[9] T. Popoviciu, Sur l'approximation des fonctions convexes d'ordre superieur, Mathematica 10, (1934), 49-54.

[10] J. M. WhitTaker, On Lidstone series and two-point expansions of analytic functions, Proc. Lond. Math. Soc., 36, (1933-1934) 451-469.

[11] D. V. WidDER, Completly convex function and Lidstone series, Trans. Am. math. soc., 51 (1942), 387-398.

[12] D. V. WIdDER, The Laplace transform, Princeton Univ. Press, New Jersey, 1941. 